\title{
Inelastic light scattering by trains of ultrashort acoustic solitons in sapphire
}

\author{
Otto L. Muskens and Jaap I. Dijkhuis* \\ Debye Institute, Department of Physics and Astronomy, University of Utrecht, P.O. Box 80 000, 3508 TA, Utrecht, The Netherlands
}

(Received 1 April 2004; published 2 September 2004)

\begin{abstract}
We monitor the development of a picosecond strain wave packet into a train of ultrashort acoustic solitons along the $c$ axis of sapphire using Brillouin scattering. One-dimensional propagation yields an intricate oscillation pattern of the scattered intensity against distance that is interpreted in terms of optical interference and Bragg resonances of light reflected from the moving soliton train. By exploring this pattern over a range of scattering angles, we derive quantitative information on the soliton parameters using only a simple analytical framework. Further more, the analogy between the wave packet and a diffraction grating is explored that provides a direct estimate of the amount of solitons in the train from the observed number of oscillation periods.
\end{abstract}

DOI: $10.1103 /$ PhysRevB.70.104301

PACS number(s): 63.20.-e, 62.30.+d

\section{INTRODUCTION}

As an extension of conventional ultrasonics into the nanometer-size regime, the method of picosecond ultrasonics ${ }^{1}$ has found wide application as an imaging tool in scientific and industrial environments. ${ }^{2-4}$ Most studies have focused on short-distance propagation in one-dimensional multilayers and in composite or nanostructured thin-films. Ballistic propagation of coherent strain packets over much longer traveling paths, however, has recently opened up a new field of fundamental and applied research. ${ }^{5-11}$ In a series of experiments in large crystals and at low temperatures, it appeared that the structure of very short strain packets becomes severely distorted by the intrinsic phonon dispersion of the crystalline lattice. ${ }^{7,8}$ Thus, it appeared that stable propagation of ultrashort strain pulses over macroscopic distances is impossible in the linear regime. However, there exists a nonlinear regime in which even shorter coherent strain pulses are formed that can travel practically without distortion.

The nonlinearity becomes apparent at intermediate strain amplitudes (typically above $10^{-4}$ ), where the pressure dependence of the sound velocity leads to self-steepening of the acoustic wave packet. In complete absence of dissipative or dispersive terms, the velocity difference between peak and the front of the packet would result in the formation of a shock-wave. ${ }^{12}$ However, the combined action of shock formation with phonon dispersion sets up a system in which stable, solitary waves are formed. ${ }^{13,14}$ Development of a picosecond strain pulse into a soliton of compression and a dispersive tail was first demonstrated using nanojoule optical pulses as an excitation source. ${ }^{9}$ In this paper, we extend the regime of strain soliton formation significantly by application of high-power (mJ) optical pulses from a $1 \mathrm{kHz}$ amplified laser system, reaching much higher strain amplitudes while covering a larger $\left(\mathrm{mm}^{2}\right)$ area of excitation. Using this setup, we recently demonstrated the breakup in a single crystal of sapphire of a picosecond strain pulse into a train of ultrashort strain solitons, ${ }^{10}$ predicting strain amplitudes of up to $0.4 \%$.

At these high strains, the nonlinear steepening process is balanced by lattice dispersion only at terahertz phonon frequencies, ${ }^{11}$ resulting in half cycle strain solitons of less than $0.5 \mathrm{ps}$ temporal width. The nonlinear development of the wavepacket turned out to be described adequately by the Kortweg-de Vries (KdV) equation,,${ }^{9,10}$ yielding estimates for the number and amplitudes of the solitons in the train. As the velocity and width of a KdV soliton depend only on its amplitude and vice versa, it is possible to draw detailed conclusions once one of these quantities has been determined with high-enough accuracy. ${ }^{15}$

In this paper we employ Brillouin scattering to determine the velocities of the strain solitons that have developed from a transducer-generated wave packet. We extend our earlier work ${ }^{10}$ to cover a range of scattering angles, providing detailed information on the relation between the Brillouin frequency and the observed spatial resonances. In this way we obtain quantitative estimates for the highest soliton velocities in the train independent of numerical simulations. Further, an alternative method of analysis of the spatial beating patterns is presented based on the analogy with an $N$-slit diffraction grating. This relates the high-frequency content to the spatial resonances at lower frequency through a scaling relation and allows for an estimation of the number of solitons.

\section{THEORY}

\section{A. KdV initial value problem}

We start our discussion with the one-dimensional nonlinear wave equation for the acoustic strain $s$, the Boussinesq equation ${ }^{12,16}$

$$
s_{t t}-c_{0}^{2} s_{z z}-\frac{\alpha}{\rho} \frac{\partial}{\partial z}\left(s s_{z}\right)-2 c_{0} \beta s_{z z z z}=0,
$$

where $\rho$ denotes the mass density, $\alpha=C_{333}+3 C_{33}$ is the nonlinearity parameter consisting of second- and third-order elasticity constants, and $\beta$ denotes the third-order correction to the LA phonon dispersion relation. In this equation, the two terms on the left describe linear propagation, the quadratic term is responsible for shock formation, and the fourth-order derivative leads to a dispersion of the propagation velocity for the different acoustic frequencies. 
Considering the experimental configuration, in which we generate a strain pulse on one side of the crystal and follow its propagation in a specific direction, it is convenient to transform to a moving frame coordinate system, defined by the parameters $t^{\prime}=t, y=z-c_{0} t$. After one integration of the resulting expression over $y$, neglecting a slow component $s_{t^{\prime} t^{\prime}}$, we arrive at the well-known Korteweg-de Vries (KdV) equation $^{13}$

$$
s_{t^{\prime}}+\frac{\alpha}{2 \rho c_{0}} s s_{y}+\beta s_{y y y}=0 .
$$

In the experimental configuration in the sapphire crystal, $\alpha<0$ and $\beta>0$, resulting in soliton development for $s<0$, i.e., for compressional strain pulses.

We are interested in the development of an arbitrary initial wave form $s(y, 0)=s_{0} \phi\left(y / l_{0}\right)$, where $s_{0}<0$ and $l_{0}$ are the typical amplitude and width of the compressional part of this initial strain packet and $\phi$ denotes the (normalized) shape of the wave packet. Therefore we choose to formulate the initial value problem in terms of the dimensionless coordinates $\eta$ $=s / s_{0}, \quad \xi=y / l_{0}$, and $\tau=t^{\prime} \beta / l_{0}^{3}$, leading to ${ }^{12}$

$$
\begin{gathered}
\eta_{\tau}+6 \sigma^{2} \eta \eta_{\xi}+\eta_{\xi \xi \xi}=0 \\
\eta(\xi, 0)=\phi(\xi)
\end{gathered}
$$

Conveniently, all the material constants and physical parameters end up in a single parameter $\sigma$ given by

$$
\sigma=l_{0}\left(\frac{\alpha s_{0}}{12 \rho c_{0} \beta}\right)^{1 / 2} .
$$

The magnitude of the similarity parameter $\sigma$ characterizes the relation between nonlinearity and dispersion in Eq. (3) and determines the number of solitons developing from the initial condition. According to Eq. (4), $\sigma$ is proportional to the area under the square root of the compressional strain wave form. In order to compare the similarity parameters for different initial value conditions, one must specify $l_{0}$ and $s_{0}$ in order to normalize the residual area of $[\phi(\xi)]^{1 / 2}$ to some predefined value, which we choose to be $\pi$, the area under the square root of the sech ${ }^{2}$-wave form. ${ }^{16}$ In the following, $\sigma$ describes this pulse "area" in units of $\pi$.

\section{B. Soliton trains}

For an arbitrary initial disturbance $\phi(\xi)$, solutions to Eq. (3) can be found using the inverse-scattering transform as the bound states of an associated scattering problem ${ }^{12,16,17}$

$$
\Psi_{\xi \xi}+\left(\lambda+\sigma^{2} \eta(\xi, \tau)\right) \Psi=0
$$

Under the initial condition $\eta(\xi, 0)=\phi(\xi)$, this equation can be solved, resulting in a complete set of scattering parameters $\Psi_{R} / \Psi_{\text {in }}$ (reflection), $\Psi_{T} / \Psi_{\text {in }}$ (transmission) and $\lambda .{ }^{18}$ It can be shown by substitution of Eq. (5) into the KdV equation (3), that the eigenvalues $\lambda$ are independent of time. This special property allows the reconstruction of the scattering potential $\eta(\xi, \tau)$ at each time for a given initial set of scattering parameters. We limit our discussion here to the most relevant result for this paper, namely the stationary states for

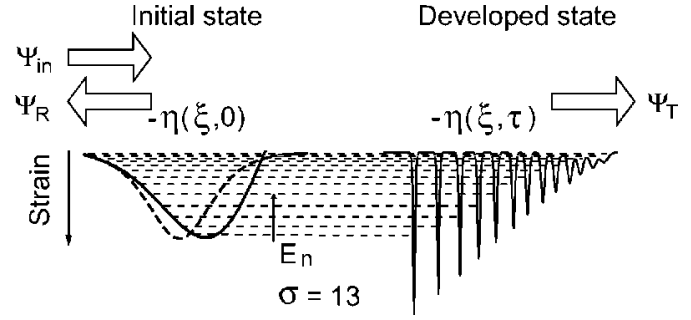

FIG. 1. Visualization of the KdV scattering problem, showing the time varying potential $-\eta(\xi, \tau)$ and the incident, reflected and transmitted wavefunctions $\Psi_{\text {in }}, \Psi_{R}$ and $\Psi_{T}$. Potentials $-\eta(\xi, 0)$ are the normalized Gaussian derivative (line) and hyperbolic secants (thick dash) functions, and the resulting soliton train for a value of $\sigma \approx 13$. Horizontal lines (dash) denote eigenvalues $E_{n}=\lambda_{n} / \sigma^{2}$ for the Gaussian derivative.

$t \rightarrow \infty$, for a discrete spectrum of eigenmodes $\lambda_{n}<0$. Figure 1 shows the typical asymptotic development of the eigenvalue problem, where each bound state $\lambda_{n}$ of the initial potential $-\eta(\xi, 0)$ corresponds to a single-soliton solution at large time $\tau$, of the form ${ }^{12,16}$

$$
\eta(\xi, \tau)=-\frac{2 \lambda_{n}}{\sigma^{2}} \operatorname{sech}^{2}\left(\left|\lambda_{n}\right|^{1 / 2}\left[\left(\xi-\xi_{0}\right)-4 \lambda_{n} \tau\right]\right),
$$

where $\xi_{0}$ denotes the variation in the position where each individual soliton splits off. In fact, all the stationary states developing from an initial compressional wave packet are solitons, completely defined by a single parameter, the eigenvalue $\lambda_{n}$. It can be observed from Eq. (6) that the amplitude of the $n$th soliton is $\eta_{n}=-2 \lambda_{n} / \sigma^{2}$, or $a_{n}=-2 \lambda_{n} s_{0} / \sigma^{2}$ in normal strain units. The velocity of these solitons in the moving frame system is $2 \eta_{n} \sigma^{2}$ in normalized coordinates, or $v_{n}$ $=\alpha a_{n} / 6 \rho c_{0}$ in real coordinates. Finally, the width is given by $\left(2 / \eta_{n} \sigma^{2}\right)^{1 / 2}$, or $l_{n}=l_{0}\left(2 s_{0} / a_{n} \sigma^{2}\right)^{1 / 2}$ in real coordinates.

An analytical solution of the eigenvalue equation (5) may be obtained in several special cases of the initial wave form $\phi(\xi)$. In particular, the spectrum of eigenvalues for a potential of the form $\phi(\xi)=\operatorname{sech}^{2} \xi$ can be found in many-quantum mechanics textbooks. ${ }^{19}$ The resulting expression for the soliton amplitudes in the train evolving from this initial wave form is given by

$$
\frac{a_{n}}{s_{0}}=\frac{1}{2 \sigma^{2}}\left(1-2 n+\sqrt{1+4 \sigma^{2}}\right)^{2} .
$$

For the combination of our experimental conditions and the material parameters of sapphire, ${ }^{9} \sigma$ takes on values in the range 5-14, and we may well approximate Eq. (7) for the leading solitons in the train to first order by

$$
a_{n} \approx 2 s_{0}(1-2 n / \sigma) .
$$

This shows that, for large $\sigma$, the first soliton in the train approaches an amplitude $a_{1} \approx 2 s_{0}$, independent of the length of the initial disturbance $l_{0}$ and of the material parameters $\alpha$ and $\beta$. The total number of bound states in this potential $\phi(\xi)$ can be determined from the condition that the term within the brackets of Eq. (7) is larger than zero, leading to an approximate number of solitons of $N \approx \sigma$ for large $\sigma$. 


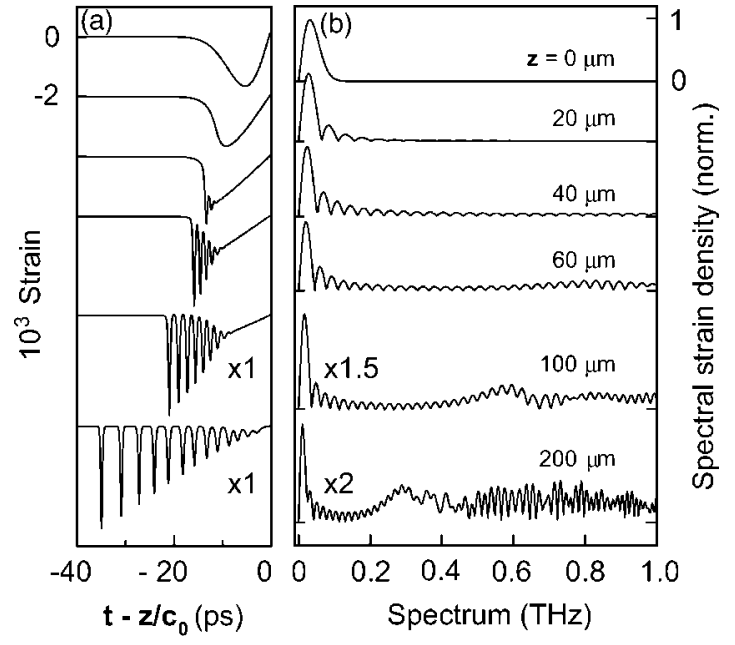

FIG. 2. (a) Simulated development of the leading, compressive part of a bipolar strain pulse during the first $200 \mu \mathrm{m}$ in a sapphire [0001] crystal, showing the initial stages of self-steepening of the wavepacket and breakup into a soliton train. (b) Development of the acoustic spectrum of the total bipolar wave packet.

In our experiments we will be dealing with bipolar strain pulses, that we choose to describe by the derivative of a Gaussian, written in dimensionless form

$$
s(y, 0)=\frac{\sqrt{2 e}}{l_{g}} s_{0} y \exp \left(-y^{2} / l_{g}^{2}\right),
$$

with an amplitude normalized to $s_{0}$ and a Gaussian waist $l_{g}$. In order to convert this to the dimensionless form $\phi(\xi)$ and the correct value of $\sigma$, the spatial dimension in Eq. (9) is normalized to the length scale $\quad \xi=y / l_{0}$ with $l_{0}$ $=e^{1 / 4} \Gamma(3 / 4) l_{g} / \pi \approx 0.5 l_{g}$, where $\Gamma$ denotes the Euler gamma function. Combined with a normalization to $s_{0}$, this gives the correct form of $\phi(\xi)$ and $\sigma$ for calculation of the number of solitons in Eq. (7).

In fact, the formula of Eq. (7) turns out to be a good approximation for soliton trains developing from our experimental pulse shapes at large values of $\sigma$. This similarity may be attributed to the strong nonlinearity, which reshapes the wave packets into the form of a shock wave before it breaks up into a series of soliton pulses. This initial developement is illustrated in Fig. 2(a) by a numerical simulation of the propagation of a compressive strain pulse formulated in the physical parameters of our experiment, ${ }^{20}$ yielding a value of $\sigma \approx 13$. Figure 2(b) shows the associated evolution of the wave packet spectrum, where formation of a shock wave generates the terahertz acoustic frequency components that have been demonstrated experimentally. ${ }^{11}$ The typical oscillations in the spectrum of the soliton train will be explained in Sec. VI B.

\section{SAMPLE AND SETUP}

In the following experiments we make use of a highpurity (less than $1 \mathrm{ppm}$ impurity ions) single-crystal piece of sapphire of dimensions $5 \times 10 \times 10 \mathrm{~mm}^{3}$. A $100 \mathrm{~nm}$ thin chromium film is evaporated onto one of the $5 \times 10 \mathrm{~mm}^{2}$ surfaces that is oriented perpendicular to the crystallographic $c$ axis. The sample is contained in an optical flow cryostat and kept at a temperature of $5 \mathrm{~K}$ during most of the experiments.

Strain pulses of high amplitude and picosecond time duration are generated thermoelastically by means of ultrafast excitation of the chromium film using femtosecond optical pulses from an amplified Ti:sapphire laser, operating at 800 $\mathrm{nm}$. For application as a transducer material, metallic films are very suitable because of their short optical absorption length and the fast response of the lattice via the electronphonon coupling. Strain wave packets are ultimately limited in width by this skin depth, which corresponds to an acoustic frequency spectrum up to several hundreds of gigahertz. ${ }^{1,21}$ However, the electronic transport and heat diffusion during the first moments after excitation ${ }^{22-24}$ allow generation of high-frequency components only in case of nanometer-thick metallic films.

Propagation of the strain wave packets through the crystal is monitored in situ using Brillouin scattering of light from a single-mode argon-ion laser, operating at $514.5 \mathrm{~nm}$. Wavevector conservation in the scattering process ensures selectivity for individual spectral components of the acoustic wave packet in the scattering process. Frequency components in the range $5-30 \mathrm{GHz}$ are accessible using a scattering configuration through the side windows of the optical cryostat. The inelastically scattered beam is frequency filtered by a quintuple-pass Fabry-Pérot interferometer and detected using a photon counting setup. Spurious background intensity is further suppressed by means of electronic time discrimination of the arriving counts. In an earlier paper, ${ }^{10}$ we investigated trains of ultrashort strain solitons using the same experimental setup. We emphasize once more that the Brillouin scattering method is well suited for the studies of strain soliton development: the propagation distance is continuously variable, the scattering wave vector matches well with the spatial walkoff of the solitons in the wave packet, and the photon counting apparatus allows for backgroundfree detection with excellent sensitivity.

\section{PUMP-PROBE CHARACTERIZATION}

Before presenting the results of the Brillouin experiments, we determine the shape of the initial strain pulses in the chromium film using pump-probe reflectometry. It is well known that nonequilibrium electron transport and thermal diffusion limit the spectral content of the strain wave form in a chromium film to less than $100 \mathrm{GHz}{ }^{22,25}$ Earlier experiments, however, have been performed at much lower laser intensities and deviations may arise at high-power excitation. To investigate the shape of the strain pulses launched into the crystal, we constructed a high-sensitivity, ultrafast pumpprobe setup based on the $1 \mathrm{kHz}$ amplified laser system, that is capable of detecting changes in reflectivity as small as 2 $\times 10^{-5}$ over a $1 \mathrm{~s}$ acquisition time. Figure 3 shows the relative reflectivity changes as a function of pump-probe delay time for the chromium film evaporated on the sapphire crystal under study, as well as for a similar film deposited onto a 


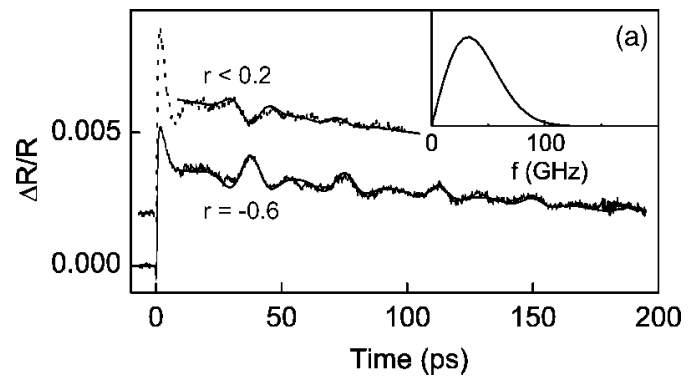

FIG. 3. Pump-probe reflectivity data of a 100-nm chromium film on lead molybdate (line) and sapphire (dash) single crystals. Smooth lines denote fits to the data. (a) Spectrum of the first acoustic echo as obtained from fits to the data.

lead molybdate sample. As the acoustic echoes from the chromium-sapphire interface are weak by the good acoustic impedance matching, we use the lead molybdate sample to determine the shape of the strain pulses generated in the chromium film. The optical response of the chromium film can be explained quantitatively using the standard theory for the elastooptic response. ${ }^{1}$ We obtain a strain profile with spectral components comparable to those measured earlier using low-amplitude excitation. ${ }^{25}$ The absolute magnitude of the reflectivity changes is almost two-orders-of-magnitude larger than what is observed in conventional picosecond ultrasonics experiments, proving the presence of large strain amplitudes. Good agreement between simulated and experimental pulse echoes is obtained for an initial strain amplitude of $4 \times 10^{-3}$ and width $l_{g} \approx 43 \mathrm{~nm}$ in the chromium film and acoustic reflection coefficients of $r \approx-0.6$ and $r<0.2$ for the lead-molybdate and sapphire substrates, respectively. After taking into account the difference in sound velocities between transducer and substrate, the strain amplitude of the pulse inside the sapphire is estimated as $2.2 \times 10^{-3}$.

\section{BRILLOUIN EXPERIMENTS}

\section{A. Polarization dependence}

For the relatively small scattering angles in the Brillouin experiment, the scattering efficiency turns out to depend strongly on the polarization angle of the light. Figure 4(a) shows a variation over three orders of magnitude of the scattered intensity for a rotation of the polarization angle $\phi$ over $90^{\circ}$. In this experiment, the scattering angle was set to $2 \theta$ $\approx 60^{\circ}$, corresponding to an acoustic frequency of $\nu_{B}$ $=22 \mathrm{GHz}$. The observed dependence corresponds favorably with the behavior expected from the decomposition of the polarization vector into its two linearly independent components $\vec{E} \perp c$ and $\vec{E} \|_{c}$ [line in Fig. 4(a)]. Using the literature values of $p_{33}=0.23$ and $p_{13} \leqslant 0.005,{ }^{26}$ a contrast of at least $2 \times 10^{3}$ is expected between both polarizations. Figure 4(a) shows a factor of 4 less contrast, which may be accounted for by some admixture of the component $p_{31} \approx 0.03$ in the elastooptic interaction, due to the nonzero angle of incidence of the probe laser. In the following, we maintain a polarization parallel to the $c$ axis to obtain an optimal scattered intensity.

\section{B. Strain calibration}

We can gauge the Brillouin spectrum induced by the coherent strain packet against the thermal phonon background

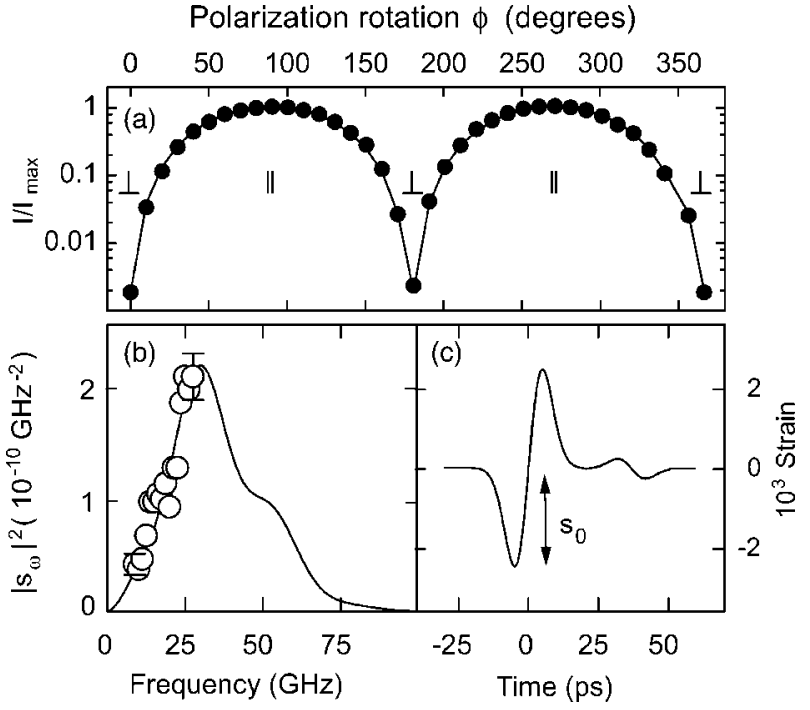

FIG. 4. (a) Dependence of Brillouin scattering intensity on the electric-field polarization angle of argon-ion laser, minimum at $\vec{E} \perp c$, maximum at $\vec{E} \| c$. (b) Power spectrum of strain waveform gauged against thermal phonon background, ○ experimental data, (line) simulation. Calibrated strain wave form obtained from (b), consistent with reflectometry data of Fig. 3.

of $\sim 40 \mathrm{cts} / \mathrm{s}$, measured in sapphire at $22 \mathrm{GHz}$ and room temperature. In the same configuration we observe a scattering yield of about $50 \mathrm{kcts} / \mathrm{s}$ for excitation with an $80 \mathrm{MHz}$, femtosecond laser at a fluence of $0.5 \mathrm{~mJ} / \mathrm{cm}^{2}$ per pulse, leading to an occupation number $n_{\omega}$ of about one-thousand times the thermally excited population.

For a harmonic oscillator, the average energy is equally distributed over the potential and kinetic parts, $U=T$ $=\frac{1}{2} \hbar \omega\left(n_{\omega}+\frac{1}{2}\right)$. Combined with the expression for the potential energy $U=\frac{1}{2} M \omega^{2}\left\langle u_{\omega}^{2}\right\rangle$, and the number of oscillators given by the Debye density of states $D(\omega) \Delta \omega$, we obtain the expression for the average wave amplitude $u_{\omega}$, given by

$$
u_{\omega}=\left(D(\omega) \Delta \omega \frac{\left(n_{\omega}+\frac{1}{2}\right) \hbar}{M \omega}\right)^{1 / 2} .
$$

We are dealing with a very directional beam of phonons, therefore, the complete, three-dimensional density of states largely overestimates the number of participating oscillators. As we gauge the occupation number $n_{\omega}$ using the thermal phonon modes selected by the acceptation angle of our interferometer system $\theta_{0} \approx 50 \mathrm{mrad}$, we should count again only this cone of wave vectors in the estimate of $u_{\omega}$. Integration over this part of phase space yields a more realistic density of states $D(\omega) \Delta \omega=V \omega^{2} \theta_{0}^{2} \Delta \omega / 4 \pi c_{0}^{3}$.

From Eq. (10) we can obtain the associated strain spectrum $s_{\omega}=\partial u_{\omega} / \partial x$ by multiplication with $\omega / c_{0}$. For our example at $22 \mathrm{GHz}$, this results in a displacement of $u_{\omega}=3$ $\times 10^{-14} \mathrm{~m}$, or an acoustic strain component $s_{\omega}=3.5 \times 10^{-7}$, over a finesse-limited bandwidth of $0.8 \mathrm{GHz}$. Calibration of the typical wave packet spectrum to this value yields a co- 


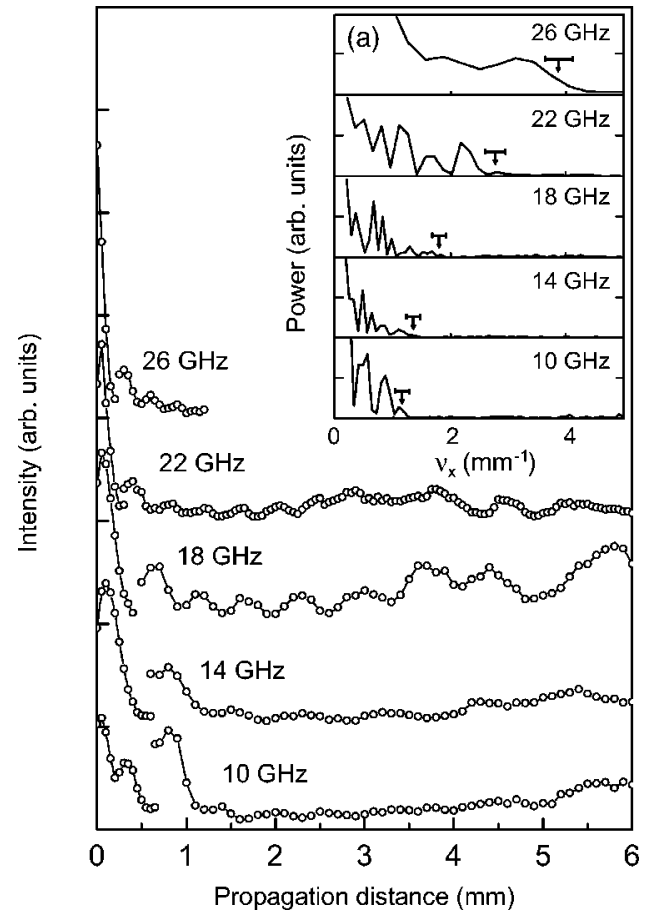

FIG. 5. Brillouin scattering intensity as a function of propagation distance at 5 different scattering angles, at a pump fluence of $6.5 \mathrm{~mJ} / \mathrm{cm}^{2}$. Inset: spatial Fourier transforms of data $(k=1 / \lambda)$, arrows indicate highest wavevector in the scans.

herent strain amplitude of $1 \times 10^{-4}$ for the "mode-locked" wave packets.

In the case of excitation by amplified laser pulses, we measured a maximum intensity of $\sim 0.5 \mathrm{kcts} / \mathrm{s}$ at $E$ $=8 \mathrm{~mJ} / \mathrm{cm}^{2}$ pump fluence. Accounting for the reduction in repetition rate, this yields a $u_{\omega}=0.7 \times 10^{-12} \mathrm{~m}$, corresponding to a coherent strain amplitude of $2.5 \times 10^{-3}$. The calibrated scale for the power density $\left|s_{\omega}\right|^{2}$ for the high-amplitude pulses is shown in Fig. 4(b) and that for the corresponding strain is shown in Fig. 4(c).

\section{Frequency dependence}

In our earlier work, we demonstrated the development of soliton trains via a direct comparison of the Brillouin scattering data with numerical simulations. An attempt was made to analyze the intricate oscillation pattern in terms of spatial resonances of the solitons, but no quantitative results were obtained using this method. Here, we attempt to get information on the soliton train using the analytical framework only, i.e., without resorting to numerical simulations. For this purpose, we have analyzed the propagation in sapphire at five selected frequency components in the range $10-26 \mathrm{GHz}$, with mutual distances of $4 \mathrm{GHz}$.

As usual, individual frequency components of the wave packet were selected by adjusting the scattering angle within the aperture limited by the size of the windows in the cryostat. At each frequency value, the development of the scattered intensity was monitored when moving the detection volume to difference positions $z$ in the crystal. Figure 5(a) shows the dependence of the Brillouin intensity on propaga- tion distance at the selected frequencies, for a pump fluence of $6.5 \mathrm{~mJ} / \mathrm{cm}^{2}$. At all selected frequencies, the scattered intensity shows a strong decrease in the first hundred micrometers of propagation, followed by an oscillatory behavior. The initial decrease can be attributed to the spectral redistribution of energy, up to terahertz acoustic frequencies, by the nonlinear steepening of the wave packet [see Fig. 2(b)] and will not be further discussed at this point. The intricate beating pattern at distances beyond the self-steepening regime can be unraveled by performing a spatial Fourier transform of the experimental traces after the initial decay, where the solitons are supposed to be well developed. The results of this procedure on the experimental traces are shown in Fig. 5(a). In all spectra we observe a distinct amount of spatial frequencies that produce the beating pattern. Unfortunately, the limited propagation distance limits the spectral resolution to $\Delta \nu_{x} \approx 0.17 \mathrm{~mm}^{-1}$, causing the resonances to overlap into broad bands. The scanning length at $26 \mathrm{GHz}$ is further limited to $1 \mathrm{~mm}$ by the size of the cryostat windows. Still, several oscillations of $\nu_{x} \approx 4 \mathrm{~mm}^{-1}$ can be observed.

The degeneracy of many of the resonances in Fig. 5(a) makes it an impossible task to assign them individually to beating modes of the soliton train. It is however possible to determine from the spectra a high-frequency cutoff, corresponding to the interference of light scattered by the fastest soliton with the light scattered by the slowest solitons and the linearly propagating tail. The estimated highest spatial frequencies for all selected scattering angles and at several pump intensities are shown in Fig. 6. We observe a linear dependence of the maximum frequency $\nu_{x}$ on the Brillouin frequency $\nu_{B}$, with a slope increasing with pump fluence. These findings are consistent with our earlier interpretation in terms of Bragg-resonances, as we will show in the next section.

\section{INTERPRETATION}

\section{A. Spatial resonances}

In the quantitative analysis of the spatial frequencies involved in the intricate oscillation patterns, we consider again a very simple model of optical interference of moving scattering objects. ${ }^{10}$ Let us consider two dielectric objects that propagate with velocities $v_{1}, v_{2}$ in the moving frame system. After a propagated distance $z$ in the crystal, the objects will have moved apart over a walk-off period $\tau$, given by $\tau$ $=\left(v_{1}-v_{2}\right) z / c_{0}^{2}$. In the Brillouin scattering experiment, one is sensitive for a resonant time delay $\tau_{\text {res }}$, given by the inverse of the Brillouin frequency $\tau_{\text {res }}=1 / \nu_{B}$. Therefore while their walk-off $\tau$ moves in and out of resonance with the probe period $\tau_{\text {res }}$, the objects will produce an oscillation in the scattered intensity with a spatial frequency $\nu_{x}$ given by

$$
\nu_{x}=\frac{\left(v_{1}-v_{2}\right) \nu_{\mathrm{B}}}{c_{0}^{2}} .
$$

This relation can now be used to obtain accurate values of the maximum velocity difference in the soliton train at different pump intensities. By fitting the experimental points of Fig. 6 using Eq. (11), [dashed lines in Fig. 6] we obtain 


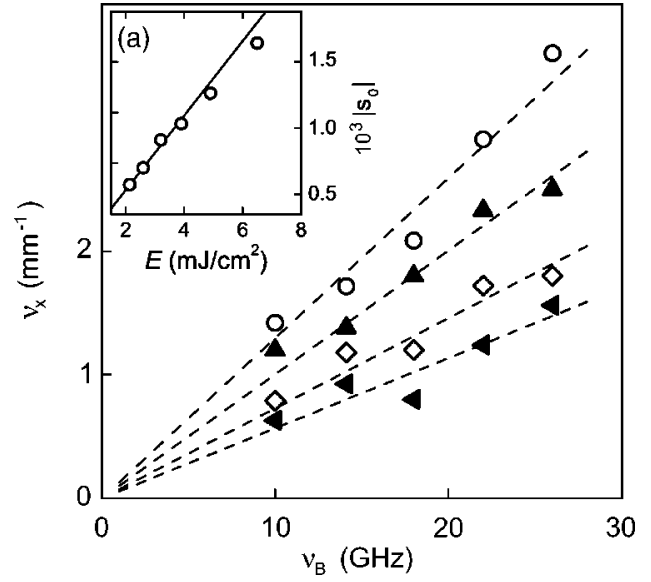

FIG. 6. Highest spatial frequency $\nu_{x}$ as a function of Brillouin frequency $\nu_{B}$ for 4 typical pump fluences $E=2.6(\varangle), 3.2(\diamond)$, $4.9(\triangle)$ and $6.5(\bigcirc) \mathrm{mJ} / \mathrm{cm}^{2}$. Lines are fits to data, following Eq. (11). Inset: initial strain amplitude $\left|s_{0}\right|$ obtained from the slope of fitted data at different pump fluences $E$. Line denotes linear dependence.

values for the velocity difference $\Delta v_{\mathrm{sol}}=v_{1}-c_{0}$ of the fastest soliton with the linear wave packet that translate directly into a maximum strain via the relations given below Eq. (6). Given the literature value of $\alpha=-1.83 \mathrm{TPa},{ }^{27}$ we find a relative soliton velocity of $\Delta v_{\text {sol }} / c_{0}=0.61\left|a_{n}\right|$ along the c axis of sapphire, where $a_{n}$ denotes the soliton amplitude. Further, from Eq. (8) we conclude that the first soliton in the train has an amplitude that is two times the initial strain amplitude $s_{0}$. Thus, the amplitude of the initial strain wave form can be obtained directly from the highest beating frequency $\nu_{x}$ in the oscillation pattern. The values for $\left|s_{0}\right|$ obtained from the linear fits are shown versus pump fluence in Fig. 6(a). Good agreement is found between these values and those obtained from detailed numerical reproduction ${ }^{10}$ of the scattered intensity at a single Brillouin frequency.

In the framework of the KdV model, velocity, amplitude, and width of a soliton are intimately connected [cf. Eq. (3)], which allows us to draw conclusions on the width and amplitude once the velocity is determined using Brillouin scattering. For the highest strain amplitude in our experiment, the amplitude of the leading soliton in the train reaches $\left|a_{1}\right|$ $\approx 3.4 \times 10^{-3}$, leading to a soliton width as small as $\tau_{1}$ $\approx l_{0} / c_{0} \sigma=0.22 \mathrm{ps}$.

\section{B. Analogy with grating}

On a related line of thought, the observed patterns in the Brillouin intensity may be considered as the orders of a diffraction grating. For this purpose, we investigated the structure of the lower-frequency part of the spectrum of the soliton trains, similar to those shown in Fig. 2(b).

We will consider exclusively the contribution of the solitons in the wave packet, as it has been shown earlier that the tail does not contribute significantly to the Brillouin scattering intensity. ${ }^{28}$ Figure 7(a) shows two soliton trains as obtained from the expressions Eq. (7) [solid line, 1] and Eq. (8) [dashed line, 2] for a value of $\sigma=11$. Note that the former of

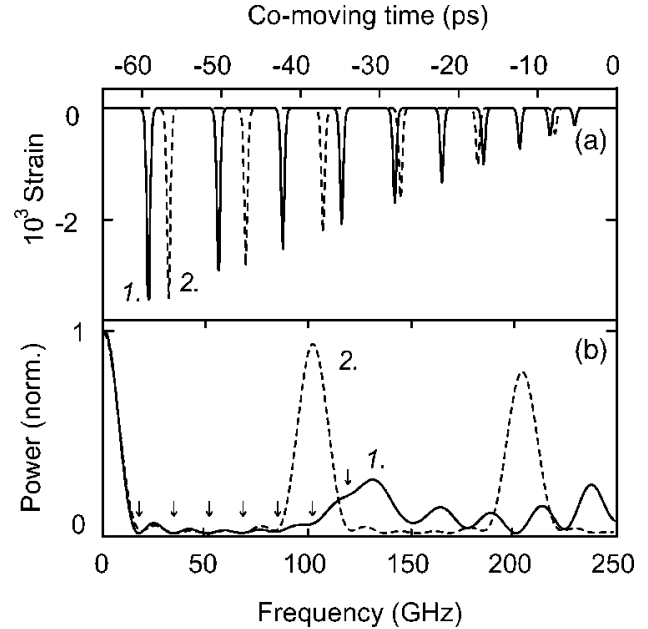

FIG. 7. (a) Analytical soliton trains after $0.5 \mathrm{~mm}$, using 1 real form, Eq. (7) (solid) and 2 equidistant approximation, Eq. (8) (dash). (b) Power spectrum of the trains 1. (solid) and 2. (dash) of (a), arrows indicate positions of local minima in the spectrum of 1 .

the two is exact, while the latter is an approximation for very large $\sigma$ that results in an equidistant spacing between the solitons. The effect of the difference in spacing for the lower part of the spectrum can be observed in Fig. 7(b). For the equidistant soliton train, [dashed line, 2] the spectrum is periodical over a range $\sim 100 \mathrm{GHz}$, while for the exact form [solid line, 1] no periodicity can be observed.

Pursuing the line of thought of our Bragg-scattering interpretation, we can analyze the spectra of Fig. 7(b) in terms of resonances between the solitons in the train. It turns out that the low-frequency spectra of Fig. 7(b) are not significantly modified when the soliton wave forms are replaced by Dirac$\delta$ functions with appropriately scaled amplitudes, therefore, we consider a train given by

$$
f(z, t)=\sum_{j=1}^{N} a_{j} \delta\left(t+\gamma_{j} z\right),
$$

with $N$ the number of solitons, $a_{j}$ the soliton amplitudes, and $\gamma_{j}=v_{j} / c_{0}^{2}$ the soliton walk-off in the moving frame system. For the equidistant train of Eq. (8), $\gamma_{j}=j \Delta \gamma$ and $a_{j}=j \Delta a$, with $\Delta a=2 s_{0} / \sigma$ the amplitude difference and $\Delta \gamma$ $=\alpha s_{0} / 3 \sigma \rho c_{0}^{3}$ the walk-off between subsequent solitons [cf. Eq. (6)]. In this case, it is useful to consider the train $f$ of Eq. (12) as a product of a comb function $g$ with a triangular envelope $h$ :

$$
\begin{gathered}
f(z, t)=g(z, t) \cdot h(z, t) \\
g(z, t)=\sum_{j=1}^{N} \delta(t+j \Delta \gamma z), \quad h(z, t)=\frac{\Delta a}{\Delta \gamma z} t .
\end{gathered}
$$

After Fourier transformation, this product will be transformed into the convolution $\tilde{f}=\widetilde{g} \otimes \widetilde{h}$, with $\widetilde{g}$ and $\tilde{h}$ the transforms of the comb and triangular functions, respectively given by 


$$
\begin{gathered}
\tilde{g}(z, \omega)=(2 \pi)^{-1 / 2} \frac{\sin N \beta \omega}{\sin \beta \omega} e^{-i \beta \omega(N+1)}, \\
\tilde{h}(z, \omega)=-i \zeta \frac{\partial}{\partial \omega} \delta(\omega),
\end{gathered}
$$

with the abbreviations $\beta=\Delta \gamma z / 2$ and $\zeta=(2 \pi)^{1 / 2} \Delta a / \Delta \gamma z$. The above expression for $\tilde{g}$ is the well-known transform function for a grating with $N$ slits, indicating that the frequency spectrum of the equidistant soliton train can be interpreted in terms of the diffraction orders of a grating. For completeness, we give the exact solution of the above convolution equation. Partial integration yields

$$
\begin{aligned}
\tilde{f}(z, \omega) & =-\left.i \zeta \frac{\partial}{\partial \omega^{\prime}} \widetilde{g}\left(\omega-\omega^{\prime}\right)\right|_{\omega^{\prime}=0} \\
& =\zeta \beta \widetilde{g}(\omega)\left(N+1+i N \frac{\cos (N \beta \omega)}{\sin (N \beta \omega)}-i \frac{\cos (\beta \omega)}{\sin (\beta \omega)}\right) .
\end{aligned}
$$

The power spectrum corresponding to this expression is shown as the dashed line in Fig. 7(b). The overall periodicity is determined by the zeros of the denominator of $\widetilde{g}$, with spacing $\Delta \omega_{0} / 2 \pi=1 / 2 \beta \approx 102.5 \mathrm{GHz}$. Further it turns out that there are exactly $(N-1)$ subminima between the main orders, with a period $\Delta \omega_{1}=\Delta \omega_{0} / N$, although their visibility is reduced by the additional terms in Eq. (15).

We are now able to understand the shape and periodicity of the spectrum of the equidistant train by considering it as a diffraction grating. It is even possible to determine the number of solitons from the fine structure in the spectrum, by counting the oscillations between the main grating orders. If we compare the periodic spectrum with that of the realistic train, shown as the solid line in Fig. 7(b), it is clear that the variations in spacing in the real soliton train are sufficient to destroy the periodicity of the grating function. Still, however, the part of the spectrum below, say $100 \mathrm{GHz}$, seems to correspond reasonably well to the pattern observed in the equidistant soliton train below the first grating order. The bump around $135 \mathrm{GHz}$ may even be attributed to constructive interference of the slightly smaller spacings in the realistic train of Fig. 7(a). Thus, by counting the number of oscillations before this maximum, we may get an impression, or lower limit, of the number of solitons in the train. For example, in Fig. 7(b) we can count up to 7 minima before the first-order maximum at $135 \mathrm{GHz}$, yielding an estimate of $N$ $=8$. This means that we underestimate the number of solitons by only two or three.
At a first glance, the spectrum of Fig. 7(b) looks very similar to the typical experimental traces for a single Fourier component, as shown in Fig. 5. This is of course the consequence of the rescaling of the spectrum over $\omega(z) \propto \omega(0) / \beta$ [cf. Eq. (14)] as the train propagates over distances $z$. This allows us to monitor the higher diffraction orders as they shift down toward the probe frequency selected by the Brillouin scattering geometry.

\section{CONCLUSIONS}

We have determined the evolution of the acoustic spectrum of a picosecond strain wave packet over millimeters of propagation at five selected Brillouin frequencies and found intricate beating patterns typical for soliton train formation. Dependence of the maximum wave vector on the selected Brillouin frequency agrees well with our interpretation in terms of Bragg resonances of the light scattered from parts of the wave packet propagating with slightly different velocities. Quantitative analysis of this dependence on the selected Brillouin frequency, for several pump fluences, provides us with accurate estimates of the strain amplitude of the first soliton in the train. These values agree well with our earlier estimates based on numerical simulations. We believe that this interpretation of the oscillations in the Brillouin intensity can be used as a direct method for estimation of the strain amplitudes in an ultrashort soliton train, without the need for carrying out detailed computer simulations.

Furthermore, via the analysis of a train of equidistant solitons we have described the low-frequency part of the solitontrain spectrum in terms of diffraction orders of a grating. The fine structure between the main grating orders in the spectrum consists of a number of oscillations that is equal to the number of solitons in the train minus one. This method of analysis of the Brillouin traces can be applied reasonably well to the realistic soliton trains in our study, despite the fact that the strict periodicity is absent by the intrinsic deviations in the distance between the solitons. The scaling of the soliton structure with propagated distance ensures that diffraction orders will shift toward lower frequencies, where they can be readily detected using Brillouin scattering. Thus, examination of the Brillouin traces yields the spectral distribution of the initial soliton train, and its fine structure allows us to determine the number of solitons in the packet.

\section{ACKNOWLEDGMENTS}

The authors wish to thank P. Jurrius and C.R. de Kok for their technical assistance. This work was supported by the Netherlands Foundation "Fundamenteel Onderzoek der Materie (FOM)" and the "Nederlandse Organisatie voor Wetenschappelijk Onderzoek (NWO)."

\footnotetext{
*Electronic address: J. I. Dijkhuis@ @ phys.uu.nl

${ }^{1}$ C. Thomsen, H. T. Grahn, H. J. Maris, and J. Tauc, Phys. Rev. B 34, 4129 (1986).

${ }^{2}$ A. Bartels, T. Dekorsy, H. Kurz, and K. Köhler, Phys. Rev. Lett.
}

82, 1044 (1999).

${ }^{3}$ O. B. Wright, B. Perrin, O. Matsuda, and V. E. Gusev, Phys. Rev. B 64, 081202 (2001).

${ }^{4}$ S. T. Huxtable, D. G. Cahill, and L. M. Phinney, J. Appl. Phys. 
95, 2102 (2004).

${ }^{5}$ J.-Y. Duquesne and B. Perrin, Phys. Rev. B 68, 134205 (2003).

${ }^{6}$ N. C. R. Holme, M. T. Myaing, and T. B. Norris, Appl. Phys. Lett. 83, 392 (2003).

${ }^{7}$ H.-Y. Hao and H. J. Maris, Phys. Rev. Lett. 84, 5556 (2000).

${ }^{8}$ H.-Y. Hao and H. J. Maris, Phys. Rev. B 63, 224301 (2001).

${ }^{9}$ H.-Y. Hao and H. J. Maris, Phys. Rev. B 64, 064302 (2001).

${ }^{10}$ O. L. Muskens and J. I. Dijkhuis, Phys. Rev. Lett. 89, 285504 (2002).

${ }^{11}$ O. L. Muskens, A. V. Akimov, and J. I. Dijkhuis, Phys. Rev. Lett. 92, 035503 (2004).

${ }^{12}$ G. B. Whitham, Linear and Nonlinear Waves, 1st ed. (Wiley, New York, 1974).

${ }^{13}$ D. J. Korteweg and G. de Vries, Philos. Mag. 39, 422 (1895).

${ }^{14}$ N. J. Zabusky and M. D. Kruskal, Phys. Rev. Lett. 15, 240 (1963).

${ }^{15}$ W. Singsomroje and H. J. Maris, Phys. Rev. B 69, 174303 (2004), determine only the velocity of the acoustic solitons using picosecond ultrasonics. To our knowledge no experimental method has yielded also the amplitude and width of an ultrashort strain soliton.

${ }^{16}$ V. I. Karpman, Non-linear Waves in Dispersive Media, 1st ed. (Pergamon Press, New York, 1975).

${ }^{17}$ C. S. Gardner, J. M. Greene, M. D. Kruskal, and R. M. Miura,
Phys. Rev. Lett. 19, 1095 (1967).

${ }^{18}$ Unlike the eigenvalues $\lambda$, which represent the amplitudes of the individual solitons in the train, the wave functions $\Psi, \Psi_{R}$, and $\Psi_{T}$ do not correspond to any physical quantity related to the experiment, but are the result of the mathematical construction.

${ }^{19}$ L. D. Landau and E. M. Lifschitz, Quantum Mechanics, Nonrelativistic Theory, 2nd ed. (Pergamon Press, New York, 1965).

${ }^{20}$ For compatibility with existing literature we present the theoretical part in the form of the initial value problem in the coordinates $(z-c t, t)$, while the Brillouin experiment is more easily understood as the equivalent boundary value problem with $(t$ $\left.-z / c_{0}, z\right)$, connected via a trivial transformation.

${ }^{21}$ C. Zhu, J. Tauc, and H. Maris, Phys. Rev. B 44, 4281 (1991).

${ }^{22}$ O. B. Wright and K. Kawashima, Phys. Rev. Lett. 69, 1668 (1992).

${ }^{23}$ G. Tas and H. J. Maris, Phys. Rev. B 49, 15046 (1994).

${ }^{24}$ O. B. Wright, Phys. Rev. B 49, 9985 (1994).

${ }^{25}$ T. Saito, O. Matsuda, and O. B. Wright, Phys. Rev. B 67, 205421 (2003).

${ }^{26}$ Landolt-Bornstein, Group III, edited by K. H. Hellwege and A. M. Hellwege (Springer, New York, 1979), Vol. 11, p. 518.

${ }^{27}$ J. M. Winey, Y. M. Gupta, and D. E. Hare, J. Appl. Phys. 90, 3109 (2001).

${ }^{28}$ O. L. Muskens and J. I. Dijkhuis, J. Lumin. 108, 297 (2004). 